\title{
FuzzingTool: Ferramenta para Testes de Intrusão em Aplicações Web
}

\author{
Vitor O. C. N. Borges ${ }^{1}$, Joaquim Q. Uchôa ${ }^{1}$ \\ ${ }^{1}$ Departamento de Computação Aplicada (DAC) \\ Instituto de Ciências Exatas e Tecnológicas (ICET) \\ Universidade Federal de Lavras (UFLA) \\ Caixa Postal 3037, CEP 37200-900 - Lavras/MG - Brazil
}

\begin{abstract}
Every day, Web applications are being used to complex activities, in order to meet market demands. With the growing use and advance of its technologies, it requires to be more concerned with information security. For that, this article presents a tool developed for intrusion testing in Web applications, the FuzzingTool. The tool uses the fuzzing technique to find flaws in these applications, with very promising results in tests, e.g., identifying several potentially unsafe auxiliary sites from a main domain.
\end{abstract}

Resumo. A cada dia as aplicações Web estão sendo usadas para atividades complexas, a fim de atender as demandas de mercado. Com o crescente uso e avanço de suas tecnologias, faz-se necessário uma maior preocupação quanto à segurança da informação. Para tal, este artigo apresenta uma ferramenta desenvolvida para testes de intrusão em aplicações Web, o FuzzingTool. A ferramenta faz uso da técnica de fuzzing para localizar falhas nessas aplicações, com resultados bastante promissores em testes, por exemplo, que permitiram identificar vários sites auxiliares, potencialmente inseguros, a partir de um domínio principal.

\section{Introdução}

Com o desenvolvimento contínuo da tecnologia Web, os navegadores, como o principal transporte de aplicativos e disseminação de conteúdo, fornecem aos usuários muitas conveniências: mecanismos de pesquisa, sites de redes sociais, compras online, transações online, etc. [Jingyu et al. 2021]. Até o início do século XXI, a maior parte de conteúdo era disponibilizado na forma de landing pages (i.e., páginas para apresentação, podendo conter formulários de contato). Atualmente, por outro lado, as páginas Web atendem parcialmente, senão completamente, toda a lógica de negócio de uma empresa, fato acelerado com a recente pandemia, em que diversas empresas optaram por mover seus negócios para aplicações Web.

Em virtude disso, manter um sistema Web seguro é imprescindível, seja por manter os serviços disponíveis e íntegros, informações sensíveis da empresa, ou pela proteção dos dados pessoais de clientes e funcionários. É importante ressaltar que as aplicações Web estão cada vez mais complexas, dinâmicas e com múltiplas funcionalidades. Diversos frameworks e tecnologias surgiram para tentar suprimir a necessidade de mercado; e, ao mesmo tempo, as vulnerabilidades acompanharam esse crescimento [Jackson 2021, Ferreira 2021].

Vulnerabilidades nas aplicações precisam ser identificadas e corrigidas para reduzir os riscos aos ativos da empresa, sendo que uma maneira bastante eficiente para essa tarefa é o uso de testes de intrusão. Em linhas gerais, um teste de intrusão visa identificar 
e, em alguns casos, explorar uma vulnerabilidade; seja para ganhar acesso total ou parcial ao sistema, obter informações sensíveis ou mesmo negar o serviço. Nas aplicações Web não é diferente, com o adicional de que, por conta de suas características, há diversas formas de se realizar um teste de intrusão.

Neste artigo será apresentada uma ferramenta voltada a testes de intrusão em aplicações Web, o FuzzingTool $]^{1}$ disponibilizado por meio da licença MIT² Antes, faremos breve introdução ao fuzzing, a seus conceitos básicos, e como essa técnica pode ser usada para identificar ou explorar vulnerabilidades nessas aplicações. Entre outras possibilidades de resultados da aplicação, tem-se, por exemplo, a identificação de sites auxiliares, potencialmente inseguros, a partir de um site principal.

\section{Conceitos Básicos}

FuzzingTool é uma ferramenta voltada a testes de intrusão em aplicações Web, utilizando fuzzing como modo de operação. Fuzzing, por sua vez é uma das técnicas utilizadas por profissionais da área de segurança da informação, inicialmente projetada para identificar crashes (i.e., interrupções inesperadas do serviço em execução) em sistemas UNIX [Miller et al. 1990]. Seu objetivo consiste em fazer requisições ao alvo por força-bruta, em formato black-box (i.e., a construção dos dados de entrada é feita sem o conhecimento prévio do alvo), enviando parâmetros totalmente ou parcialmente malformados para a aplicação [Forrester and Miller 2000] ]. Ao realizar tais requisições, a aplicação pode responder de diversas maneiras, incluindo exceções ou mesmo com informações sensíveis sobre o alvo, ou mesmo interrompendo o seu serviço.

\section{A simplicidade desta técnica significa que ela tem um baixo custo e é fácil de se aplicar [...]. Nosso artigo de 1990 observou que a única falha de maior categoria no dia era o acesso fora dos limites de um buffer e sugeriu que o teste de difusão poderia ser usado [...] para ajudar a encontrar fa- lhas de segurança”. Por mais simples que seja, ainda é o tipo de erro que continua a causar interrupções massivas na Internet, conforme evidenci- ado pela vulnerabilidade Heartbleed no OpenSSL em 2014 e a vulnerabili- dade mais recente no clássico comando sudo em 2018 [Miller et al. 2020].}

Do ponto de vista da Web, essa técnica pode ser aplicada a uma infinidade de cenários. Requisições Web carregam consigo, obrigatoriamente, um cabeçalho e, em alguns casos, também carregam um corpo; as respostas a tais requisições funcionam da mesma forma, com a diferença de que quase sempre trazem um corpo. Enquanto o corpo da requisição serve para enviar dados ao servidor, o corpo da resposta contém a própria página buscada. Entender como essas requisições são feitas, e como são respondidas, se torna o ponto chave para se tirar maior proveito da técnica de fuzzing.

Os vetores de ataque mais comuns utilizados por essa técnica são os campos de formulários e parâmetros de requisições GET. No geral, procuram-se vulnerabilidades como SQL Injection, XSS (i.e., Cross-Site Scripting) e path traversal, listadas entre as maiores vulnerabilidades Web [The OWASP Foundation 2020]. Contudo a abordagem não se limita a apenas tais vetores, podendo também ser aplicada à enumeração dos métodos aceitos pela requisição, à enumeração dos caminhos da requisição (arquivos e diretórios), e à injeção de código nos cabeçalhos (e.g., dentro dos cookies).

\footnotetext{
${ }^{1}$ FuzzingTool: https://github.com/NESCAU-UFLA/FuzzingTool

${ }^{2}$ Licença utilizada: https://github.com/NESCAU-UFLA/Fuzzing Tool/blob/master/LICENSE
} 
Pela sua natureza de ser um método exaustivo, muita informação é gerada; separar apenas as respostas de interesse é fundamental para se evitar falsos positivos. Entram no escopo da análise, dependendo do que se está testando, as seguintes informações: o código de status, latência, tamanho do corpo, conteúdo do corpo, URL de destino, redirecionamentos e cabeçalho. Por exemplo, ao procurar por vulnerabilidades de CrossSite Scripting, deve-se investigar no corpo da resposta se o payload refletiu integralmente, ou seja, sem que tenha ocorrido nenhuma remoção ou escape de caracteres especiais.

Dentre as ferramentas gratuitas de fuzzing para aplicações Web, existem várias aplicações similares, entretanto, são poucas aquelas que tenham sido favoritadas por pelo menos 1000 usuários no Github, com recursos para modificar parâmetros de ataque e adicionar opções de requisição e afins. Das que atendem esses critérios, foram identificadas apenas as seguintes aplicações: Wfuzz ${ }^{3}$ e Ffuf $f^{4}$ que, assim como a ferramenta apresentada neste trabalho, prestam funcionalidades gerais de fuzzing; dirsearch $h^{5}$ para enumeração de arquivos e diretórios; Gobuster ${ }^{6}$ para enumeração de hosts virtuais, DNS e arquivos e diretórios. O diferencial do FuzzingTool para as ferramentas mencionadas é sua capacidade de construir o resultado de acordo com o tipo de ataque feito, além da apresentação desses resultados no terminal. Por exemplo, ao se realizar uma enumeração de subdomínios, o endereço IP do alvo é adicionado ao resultado. Desse modo podemos ter resultados com pontos específicos de interesse, sem perder dados importantes sobre os testes feitos.

\section{A Ferramenta}

FuzzingTool foi desenvolvido em Python 3.6, para uso em ambientes Linux, por meio de interface de linha de comando. Para o desenvolvimento, foi utilizada orientação a objetos e alguns padrões de projeto, como decorator, factory e strategy [Refactoring Guru 2014]. Inicialmente foi projetada apenas para testes de Cross-Site Scripting e SQL Injection em aplicações Web locais, de maneira sequencial. Atualmente, ela permite of fuzzing de dados (corpo e parâmetros da requisição), enumeração dos métodos da requisição, enumeração dos arquivos e diretórios existentes na aplicação, e enumeração dos subdomínios do alvo que contenham um servidor Web ativo, além de realizar requisições em paralelo. $\mathrm{Na}$ Figura 1, é apresentado seu modo de funcionamento.

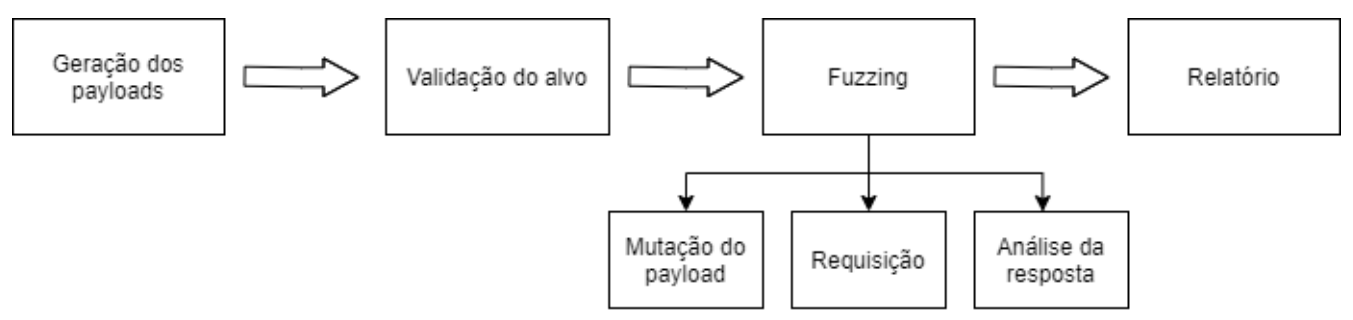

Figura 1. Modo de funcionamento

Quando da chamada da aplicação, inicialmente é verificado se todos os argumentos foram instanciados corretamente. Após, ocorre o processo de geração dos payloads, encarregado por gerá-los e também definir suas regras de mutação. Antes de iniciar o fuzzing, o alvo em questão é validado ao realizar um teste de conexão (e redirecionamentos

\footnotetext{
${ }^{3}$ Wfuzz: https://wfuzz.readthedocs.io/en/latest/

${ }^{4} \mathrm{Ffuf}:$ https://github.com/ttut/ttut

${ }^{5}$ dirsearch: https://github.com/maurosoria/dirsearch

${ }^{6}$ Gobuster: https://github.com/OJ/gobuster
} 
para o caso de fuzzing de dados). Já na fase de fuzzing é onde se encontra o núcleo da ferramenta, dividindo-se em três partes: a mutação do payload, a requisição e a análise da resposta. Por fim há a etapa de escrita dos dados obtidos num relatório.

Na etapa de geração dos payloads, é criado um objeto Dictionary, globalmente ou para cada alvo, contendo uma ou mais wordlists. Tais wordlists podem ser geradas a partir de um arquivo, uma lista de palavras ou mesmo geradas por plugins. Esse objeto Dictionary utiliza os métodos estáticos de uma outra classe denominada Payloader, responsável por realizar a mutação dos payloads durante a fase de fuzzing. Esse Payloader detém as configurações de prefixos, sufixos, caixa alta e baixa, e dos encoders. O conteúdo das wordlists é inserido numa fila, que será consumida pelo processamento mais adiante.

$\mathrm{Na}$ fase de fuzzing, ocorre o processamento do programa. O Fuzzer - objeto responsável pelo fuzzing - consome a fila de payloads do Dictionary (com as regras de mutação), enviando essa carga à requisição e, ao receber uma resposta, ela é tratada num objeto Resultado, para então ocorrer a sua análise. Esse resultado por sua vez contém informações básicas, presentes em todos os tipos de testes: o payload utilizado na requisição, a URL do alvo, o tamanho do corpo da resposta (em bytes), a quantidade de linhas e palavras no corpo da resposta e a latência.

A análise é feita em duas partes aninhadas (i.e., para que a segunda análise seja feita, ela depende do resultado da primeira), com a intenção de diminuir falsos positivos. À primeira instância, é verificado pelo objeto Matcher se um conjunto de regras estabelecidas pelo usuário se aplicam ao resultado, tais como: códigos de status HTTP, tamanho do corpo da resposta (em bytes) e a latência. Em sequência o objeto Scanner, escolhido automaticamente pelo programa ou por um plugin, pode ou não efetuar uma avaliação em cima do resultado.

Cada Scanner, além de fazer essa avaliação adicional, faz ao menos uma adição de informação ao objeto Resultado, e define o callback para a escrita desse resultado à interface de saída. O PathScanner, um dos scanners padrões da ferramenta, adiciona a informação da URL de redirecionamento nos casos em que o código de status 301 e 302 são detectados. No callback de escrita, ele faz a coloração dos códigos de status para facilitar a identificação de páginas com acesso negado, acessíveis ou com redirecionamentos.

Existem alguns recursos para desvio de firewall e WAF (Web Application Firewall) [Yari et al. 2019], tais como: alterar os cabeçalhos da requisição por completo (ao invés de passar uma URL como alvo para a ferramenta, é passado um arquivo que contenha as suas informações de requisição), fazer a mutação do payload, definir um delay entre cada requisição, e usar proxies.

Há a opção de colocar códigos de status HTTP em uma lista negra. Com isso, é possível que o programa execute uma ação específica ao detectá-los, como: pular o alvo atual ou pausar a execução por um período de tempo. Tal funcionalidade se torna interessante quando, por exemplo, queremos evitar a sobrecarga do alvo ao colocar na lista o código 500 (Internal Server Error); ou mesmo quando queremos pausar a ferramenta caso o alvo venha a rejeitar requisições, colocando na lista o código 429 (Too Many Requests).

O aplicativo dispõe de mais de 30 argumentos a serem usados nos testes, sendo os principais o -u para especificar o alvo e o -w para especificar wordlists. Outras opções que merecem ser listadas são o -X para definir o método da requisição e o -t para definir a quantidade de threads que serão usadas. Para uma completa verificação dos argumentos disponíveis, pode-se usar o opção - $h$ ou -help. 


\section{Testes e Resultados}

Para uma melhor análise da ferramenta, foram realizados três testes, dois deles realizados em um ambiente providenciado propriamente para testes de intrusã $\mathrm{q}^{7}$. O primeiro teste foi realizado para identificar vulnerabilidades de SQL Injection [Sivasangari et al. 2021] em uma página de login. Nesse teste, foi realizado fuzzing de dados e, quando um comparador de dados não é especificado ao Matcher, o próprio programa tenta calcular e sugerir ao usuário as informações para este comparador. Esse cálculo é feito a partir de uma requisição à página alvo, coletando informações como o tamanho do corpo da resposta em bytes, e a latência dela. A wordlist utilizada foi construída objetivamente para testes gerais de SQL Injection, contendo 1358 payloads.

Como pode ser verificado na Tabela 1 , foram encontrados 22 resultados para o ataque Boolean-Based que atenderam às regras do Matcher. Em contrapartida, os testes para o Time-Based tiveram resultados discrepantes ao variarmos os valores da configuração do Matcher. Isso se dá pois a latência de uma requisição Web pode variar de acordo com o estado da conexão tanto do cliente quanto do servidor. Por esse motivo, ajustar a configuração para atender a uma latência maior trará, dependendo da wordlist, alguns falsos negativos, porém, com uma maior segurança.

Tabela 1. Resultados para o teste de SQL Injection

\begin{tabular}{|c|c|c|c|}
\hline $\begin{array}{c}\text { Tipo de ataque } \\
\text { Blind } S Q L i\end{array}$ & $\begin{array}{c}\text { Configuração do } \\
\text { Matcher }\end{array}$ & $\begin{array}{c}\text { Resultados } \\
\text { encontrados }\end{array}$ & Falsos-positivos \\
\hline Boolean-Based & $\begin{array}{c}\text { Tamanho do corpo } \\
\text { da resposta }>5823 \\
\text { bytes }\end{array}$ & 22 & $0 \%$ \\
\hline Time-Based & Latência $>2$ seg & 73 & $97,26 \%$ \\
\hline Time-Based & Latência $>10$ seg & 1 & $0 \%$ \\
\hline
\end{tabular}

O segundo teste foi feito para enumerar arquivos e diretórios da aplicação. A wordlist utilizada continha 4780 payloads, com arquivos que, normalmente, não deveriam estar expostos, como: arquivos de configuração do Apache, do WordPress e de projetos do Github. Pela natureza do teste, deseja-se encontrar os arquivos e diretórios existentes que são normalmente acessíveis, os que levam a redirecionamentos, e os que têm acesso restrito. Para isso foram incluídos os códigos de status 200, 301, 302, 401 e 403 nas regras do Matcher. Foram encontradas 19 páginas com status 200, duas páginas com status 301 e uma com status 403.

O terceiro teste consistiu em enumerar hosts (subdomínios) que teriam o serviço HTTP ativo, de duas instituições federais de ensino superior. Em cada alvo foi utilizado o plugin DnsZone para a construção da sua respectiva wordlist, obtendo os payloads para o teste a partir da exploração da vulnerabilidade de transferência de zona irrestrita [CVE 1999]. Na primeira instituição, foi gerada uma wordlist com 993 payloads, encontrando 151 hosts com o serviço ativo. Para a segunda instituição, foi gerada uma wordlist com 453 payloads, encontrando 63 hosts com o serviço ativo.

\footnotetext{
${ }^{7}$ Acunetix Acuart: http://testphp.vulnweb.com/
} 
Com os resultados obtidos, percebe-se o potencial da ferramenta. No caso das instituições de ensino, por exemplo, geralmente é considerado que o site principal é relativamente vigiado e seguro. Entretanto, os sites auxiliares geralmente não recebem a devida atenção, sendo alvo fácil de ataque, possibilitando a escalada de acesso a recursos computacionais das instituições.

\section{Conclusão}

Dado o crescente desenvolvimento das tecnologias em aplicações Web, torna-se cada vez mais importante se preocupar quanto à sua segurança. Neste artigo foi apresentado como a técnica de fuzzing é aplicada para testes de intrusão nessas aplicações, com o foco em uma ferramenta para lidar com esses testes, listando seus modos de operação e um exemplo de uso.

Para trabalhos futuros, alguns já em andamento, pretende-se: adicionar uma interface Web à ferramenta, utilizando a biblioteca Flask do Python; adicionar mutadores dos payloads voltados à linguagens específicas (e.g., SQL, JavaScript); adicionar novos encoders (e.g., um para tratar códigos hexadecimais em HTML, denominado HtmlHexadecimal); adicionar um mecanismo de detecção e prevenção a honeypots; inserir a ferramenta no repositório oficial do Kali Linux ${ }^{8}$.

\section{Referências}

CVE (1999). CVE-1999-0532. Disponível em: https://cve.mitre.org/cgi-bin/cvename.cgi?name= 1999-0532. Acesso em: 06 de Julho, 2021.

Ferreira, N. (2021). Joomla JCK Editor 6.4.4 - 'parent' SQL Injection (2). Disponível em: https: //www.exploit-db.com/exploits/49627, Acesso em: 06 de Julho, 2021.

Forrester, J. E. and Miller, B. P. (2000). An empirical study of the robustness of Windows NT applications using random testing. 4th USENIX Windows Systems Symposium.

Jackson, T. (2021). Wordpress Plugin YOP Polls 6.2.7 - Stored Cross Site Scripting (XSS). Disponível em: https://www.exploit-db.com/exploits/50066. Acesso em: 06 de Julho, 2021.

Jingyu, Z., Hongchao, H., Shumin, H., and Huanruo, L. (2021). A XSS attack detection method based on subsequence matching algorithm. In 2021 IEEE International Conference on Artificial Intelligence and Industrial Design (AIID), pages 83-86.

Miller, B., Zhang, M., and Heymann, E. (2020). The relevance of classic fuzz testing: Have we solved this one? IEEE Transactions on Software Engineering, pages 1-1.

Miller, B. P., Fredriksen, L., and So, B. (1990). An empirical study of the reliability of UNIX utilities. Communications of the ACM, (33).

Refactoring Guru (2014). Padrões de projeto em python. Disponível em: https://refactoring.guru/ pt-br/design-patterns/python. Acesso em: 06 de Julho, 2021.

Sivasangari, A., Jyotsna, J., and Pravalika, K. (2021). SQL injection attack detection using machine learning algorithm. In 2021 5th International Conference on Trends in Electronics and Informatics (ICOEI), pages 1166-1169.

The OWASP Foundation (2020). Top 10 web application security risks. Disponível em: https: //owasp.org/www-project-top-ten/. Acesso em: 06 de Julho, 2021.

Yari, I. A., Abdullahi, B., and Adeshina, S. A. (2019). Towards a framework of configuring and evaluating modsecurity waf on tomcat and apache web servers. In 2019 15th International Conference on Electronics, Computer and Computation (ICECCO), pages 1-7.

\footnotetext{
${ }^{8}$ Kali Linux: https://www.kali.org/
} 